\title{
Overcoming Barriers to Accessing Surgery and Rehabilitation in Low and Middle-Income Countries: An Innovative Model of Patient Navigation in Nepal
}

\author{
Jennifer L. Ibbotson ${ }^{1} \cdot$ Bijata Luitel $^{2} \cdot$ Bikash Adhikari $^{2} \cdot$ Kathryn R. Jagt $^{1} \cdot$ Erik Bohler $^{3} \cdot$ \\ Robert Riviello $^{4} \cdot$ Geoffrey C. Ibbotson $^{5}$
}

\begin{abstract}
Background Injury and disability are prominent public health concerns, globally and in the country of Nepal. Lack of locally available medical infrastructure, socioeconomic barriers, social marginalization, poor health literacy, and cultural barriers prevent patients from accessing surgical and rehabilitative care. Overcoming these barriers is an insurmountable challenge for the most vulnerable and marginalized, resulting in absence of treatment or even death. Methods Sundar Dhoka Saathi Sewa (SDSS), a non-government organization, provides a patient navigation service which facilitates referrals to tertiary centers from Nepal's most remote areas. Specific criteria ensure that patient referrals are appropriate in regard to clinical and socioeconomic need, while comprehensive counselling helps guide the patient and family. The SDSS staff meet patients upon arrival in Kathmandu and facilitate admission to the appropriate tertiary hospital. They advocate for the patient, provide medicine, supply food and cover all treatment costs.

Results This project has enabled access to treatment for more than 1200 children for conditions leading to long-term disability and/or congenital heart disease. Interventions include a wide range of surgical and rehabilitative procedures such as complex orthopedics, cleft lip and palate, congenital talipes equinovarus, burn contractures, neurological cases, and cardiac surgery for valvular disease, septal defects and other congenital malformations.

Discussion The SDSS model of patient navigation is effective in overcoming the barriers to access surgical care and rehabilitation in Nepal. The success is owed to committed international donors, capacity building, effective counselling, advocacy, compassion, and community. We believe that this model could be replicated in other LMICs.
\end{abstract}

Geoffrey C. Ibbotson

geoff.ibbotson@unitar.org

1 Samaritan's Purse Canada, 20 Hopewell Way NE, Calgary, $\mathrm{AB}$, Canada

2 Sundar Dhoka Saathi Sewa, Bagdole - 4, PO Box 8975, EPC1961, Lalitpur, Nepal

3 University of Oslo, Åsterudvn. 30, 1344 Haslum, Norway

4 Department of Global Health and Social Medicine, Massachusetts Center for Surgery and Public Health, Brigham and Women's Hospital, Harvard Medical School, Boston, MA, USA
5 United Nations Institute for Training and Research (UNITAR), Palais des Nations, 1211, Geneva 10, Switzerland 


\section{Background}

The World Health Organisation (WHO) recognizes that injury and disability are prominent global public health concerns. Traumatic injury alone causes more fatalities than malaria, TB and HIV combined [1, 2]. Of those who survive their injuries, many then suffer from significant disability as a direct result of delayed, non-existent or inadequate access to surgical management and rehabilitation care.

More than 1 billion people live with some form of disability [1,3], with nearly 200 million experiencing considerable difficulties in functioning [1, 4]. The vast majority reside in low- and middle-income countries (LMIC), where disability is a key cause and consequence of poverty [3-5].

The United Nations Sustainable Development Goals (SDGs) promote health as a primary focus in Goal 3, "Ensure healthy lives and promote well-being for all at all ages" [6], which is closely linked to Goal 1: "End poverty in all its forms everywhere". In order to achieve this, access to surgical care and rehabilitation must become a higher priority [7, 8]. The World Bank, the Lancet Commission on Global Surgery, and the United Nations have called upon all countries to ensure that no one is forced into poverty due to the costs of medical care [7-10].

Multiple barriers prevent patients in LMICs from accessing treatment: lack of locally available medical infrastructure; equipment; and clinical expertise; socioeconomic barriers; social marginalization; poor health literacy; and cultural barriers $[3,4,11]$.

The country of Nepal faces significant challenges being ranked as one of the poorest countries in Asia (Human Development Index score 144/188). Its remote communities are considered some of the most vulnerable in the world [12-14]. With over 28 million people in an area covering $147,181 \mathrm{~km}^{2}$, its population monograph recognizes 123 languages and more than 100 people groups [15], stratified into 125 identified castes [16].

Prior to 1950, Nepal was completely isolated from the outside world. The government has made great strides forward in improving healthcare since then. Today, they are working to deliver Basic Healthcare Services in an effort to reach Universal Health Coverage [17].

Presently, Nepal has eight central tertiary care hospitals, 12 regional level hospitals, 70 district level hospitals, nearly 13,000 primary healthcare outreach clinics, two rehabilitation centres, and one specialized Hospital and Rehabilitation Centre for Disabled Children (HRDC) $[18,19]$. Although Nepal's medical colleges graduate over 2000 physicians and 500 specialists annually, the majority remain in urban centres [20]. The availability of a surgeon at the district hospitals is variable with the remote facilities facing regular absenteeism [21].

In remote locations where advanced surgery or rehabilitation services are not available, the only option for receiving care is referral to Kathmandu [22-25].

Having worked for decades in isolated clinical settings, our team of clinicians and managers experienced repeated failure when referring patients because the most marginalized and vulnerable were unable to overcome the aforementioned barriers, resulting in the absence of treatment, sometimes even death.

\section{Method}

In 2005, an indigenous Nepali church, whose many persons with disabilities knew first-hand the challenges of accessing healthcare in Kathmandu, initiated a comprehensive and integrated patient navigation service to overcome the barriers they had experienced. As a result of this work, a non-government organization called Sundar Dhoka Saathi Sewa (SDSS, Beautiful Gate Friend Service) was established in 2008.

SDSS identifies and provides solutions for accessing healthcare for vulnerable patients from Nepal's most remote areas. For the purposes of this paper, we will specifically describe the Child Heart and Rehabilitation Care Project (CHRCP) from 2006 to 2018, hereafter called "the project", one of several projects that SDSS manages.

The project's initial goal was to enable remote hospitals and clinics to accept complex pediatric cases who require long-term rehabilitation, including surgical interventions, by giving them access to referral to a tertiary centre.

A year later, the criteria were expanded to include patients who required heart surgery for congenital or rheumatic heart disease. This part of the project integrates into the Government of Nepal's free cardiac care program.

Additionally to navigating the patients through the referral process, the project provides funding for travel, food, and lodging for the patient and one caregiver. It offers financial coverage for rehabilitative surgery, orthotics, prosthetics, physical therapy, occupational therapy, speech and language pathology, casting, assistive devices, and pre-operative investigations. The financial support spans the entire referral process from the sending facility to the receiving facility and the child's return home. Approximately $95 \%$ of the patients referred are considered extremely vulnerable and require full financial assistance. Funding from an international organisation has been constant since 2006, while always seeking to empower Nepali families to use their own finances whenever possible. 
Table 1 Project acceptance criteria

Acceptance criteria

1) Clear diagnosis of a physical disability or heart defect

2) Patient appropriate for extensive rehabilitation and/or surgical intervention

3) Patient under the age of 18

4) Completion of a standardized clinical assessment form by an approved rehabilitation therapist or physician confirming appropriateness of referral

5) Completion of a standardized socioeconomic assessment form by the sending hospital social services department, confirming socioeconomic need

The acceptance criteria are dependent on the patient's condition with regard to diagnosis, appropriateness and age (1-3). They are dependent on the sending clinic with regard to assessment by a licensed rehabilitation therapist or physician, as well as assessment by social services (4-5)

Table 2 A simple, step-by-step protocol for the referral of rehabilitation patients

\section{Sending facility}

Rehabilitation therapists and/or physicians determine the need for long-term rehabilitation therapy

- Complete clinical assessment form

Social services evaluate socioeconomic need

- Complete social assessment

- Contact the patient navigation centre by phone to initiate referral

- Arrange transportation, food and lodging for one overnight in the bazaar (local town) if necessary

- Communicate patient's name, diagnosis and arrival time to the centre and to the rehabilitation coordinator via e-mail

\section{SDSS patient navigation}

Patient navigation (PN) staff meet patient and caregiver at the arranged arrival location (bus park, airport)

PN staff take patient and caregiver to the centre or directly to the hospital when urgent admission is needed:

- Provide food and lodging

PN staff counsel the patient and family, requesting consent prior to admitting the patient into the project

PN staff accompany the patient to the hospital on the scheduled admission date:

- Facilitate admission

- Advocate for treatment

- Assist in purchasing medicine and materials

- Arrange inpatient food provision

PN staff visit regularly during admission

PN staff assist with discharge from the receiving facility

Healthcare coordinator evaluates patient's mobility post-treatment, writes a discharge summary and sends it to the sending facility

PN staff arrange for follow-up treatment

PN staff arrange return travel for patient and caregiver and coordinate adaptations to home environment, when indicated

\section{Project criteria}

Specific inclusion criteria ensure that patient referrals are appropriate with regard to clinical and socioeconomic need (Table 1). These criteria have played a critical role in the success of the project by defining the target patient groups and focussing the limited funding and manpower on specific clinical diagnoses with measurable outcomes.

To build sustainability, physicians, rehabilitation therapists and social workers at the sending facilities refer patients directly to the patient navigation centre at SDSS using a simple, step-by-step protocol (Table 2), which they have been trained to complete. Pediatricians skilled in cardiac ultrasound techniques hold the sole authority to complete the cardiac assessment.

We chose sending facilities based on their remote location, a proven track record of providing high quality healthcare to the most marginalized people groups and their non-refusal policy. 


\section{Referral process}

Both the medical team and social services at the sending facility provide comprehensive counselling to the patient and family to explain the reason for the referral, including the estimated length of treatment and the expected outcomes. The social services staff contact the SDSS patient navigation coordinator in Kathmandu to authorize the referral.

The patient navigation staff meet the patient and accompanying family member as soon as they arrive in Kathmandu and transport them either to the patient navigation centre or directly to the hospital if necessary (Fig. 1).

Patient navigators facilitate the process of hospital admission, advocate for appropriate attention from the medical staff, and counsel the patient and family. They assist the hospital staff with obtaining consent for treatment, purchase any medicines and materials required, supply nutritious food, and cover all costs associated to treatment. They regularly visit the patient throughout their admission and coordinate their discharge from the hospital. If the treatment includes outpatient care, they provide lodging, food and transport through the patient navigation centre. Finally, they organize the patient's return home, educating the patient about follow-up visits and the care plan.

\section{Results}

During the initial years, patient numbers started small, with only 17 patients referred in 2006-07. Referrals increased progressively during the following five years.

The annual number of patients showed a sharp increase in early 2013 and again in early 2017 (Fig. 2). The first rise corresponded to the opening of the new patient navigation centre in Kathmandu, which increased the patient capacity of the project from 25 to 40 in-patients.

The second increase in 2016-17 corresponded to SDSS hosting a partner organization conference that brought together all the sending facilities and receiving tertiary centres. Clarifying criteria gave partners confidence to effectively focus their referrals.

Referrals increased when sending facilities developed their diagnostic abilities. Conversely, referrals dropped when sending facilities increased their capacity for treatment, for example, by hiring a physical therapist or an orthopedic surgeon, enabling patients to access treatment locally.

The rehabilitation component of the project received patients with burn contractures; cleft lip and/or palate; congenital talipes equinovarus (CTEV); complex orthopaedics; conditions requiring amputations; neurological disorders, and spinal cord injuries. Children with cardiac conditions presented with atrial and ventricular septal defects; valvular disease, complications of rheumatic heart disease; and congential anatomical abnormalities such as tetralogy of Fallot, Ebstein's anomaly, patent ductus arteriosis, transposition of the great arteries, and truncus arteriosis (Fig. 3).

The rehabilitation surgical interventions included open reduction internal fixation (ORIF); external fixation; corrective osteotomy; amputation; stump revision; wound debridement; skin grafts; post-burn contracture release; heel cord release; tendo-Achilles lengthening; and spinal surgery including laminectomy and vertebral fusion. Ponsetti serial casting followed by splinting and orthotics was widely used for CTEV cases. All rehabilitation patients received comprehensive physical therapy, occupational therapy, and speech and language pathology.

The cardiac surgical interventions included septal defect closures, PTFE patch catheterization, intra-cardiac repair of tetralogy of Fallot, multiple techniques for valve repair and valve replacement.

These examples illustrate the complexity of surgical care accessed through the project, which would have otherwise been inaccessible (Table 3). Patients were often in critical condition due to extreme delays in diagnosis and treatment.

The typical length of stay at SDSS in 2017-18 for patients coming for rehabilitation medicine was 40 days, compared to 13 days for heart patients.

The project began with three patient navigators. It has now grown to involve 32 staff, of whom 18 are directly engaged in patient navigation.

Children from 38 of Nepal's 77 districts came through the project (Fig. 4) with ages ranging from newborn to 18 years (Fig. 5).

\section{Discussion}

We have found this model of patient navigation to be effective in overcoming the multiple barriers to access surgical care and rehabilitation in Nepal. As to be expected, this project faced a number of challenges (Table 4).

The following features contribute to reaching the goal of overcoming barriers: 

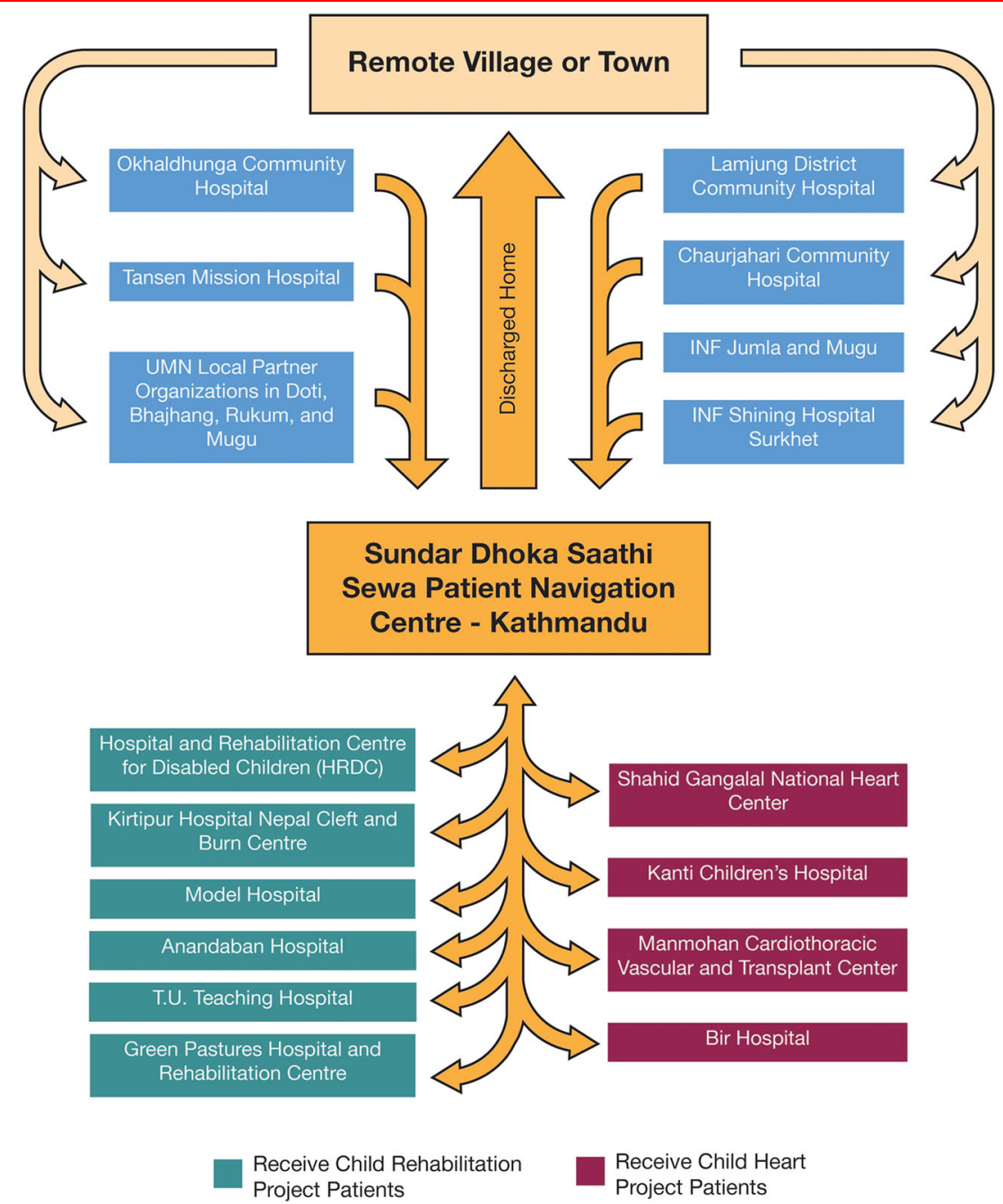

Fig. 1 Patient flow chart

$$
250
$$

200



Fig. 2 Annual number of CHRCP patients 
Table 3 Number of patients per treatment category

\begin{tabular}{|c|c|}
\hline CHP & \\
\hline Septal defect surgery & 42 \\
\hline Surgery for other anatomical abnormalities & 42 \\
\hline Valve surgery & 30 \\
\hline Cardiac catheterization (diagnostic) & 15 \\
\hline Medical treatment & 298 \\
\hline CRP orthopedic surgery & \\
\hline ORIF & 57 \\
\hline Corrective osteotomy, bone graft, sequestrectomy & 31 \\
\hline External fixator & 22 \\
\hline Amputation, stump revision & 21 \\
\hline Joint surgery (ankylosis release, arthrodesis, arthroplasty) & 11 \\
\hline Alveolar bone graft & 7 \\
\hline Skeletal traction & 6 \\
\hline Implant surgery & 5 \\
\hline Spinal surgery & 2 \\
\hline CRP soft tissue surgery & \\
\hline Heel cord release & 63 \\
\hline Contracture release & 42 \\
\hline Skin grafts (FTSG, STSG) & 32 \\
\hline Surgical wound debridement, incision and drainage of abscess & 29 \\
\hline Reconstructive surgery & 25 \\
\hline Cleft lip repair & 23 \\
\hline Palatoplasty & 20 \\
\hline Tendo-Achilles lengthening & 13 \\
\hline Neurological (craniectomy, myelomeningocele reduction) & 5 \\
\hline CRP conservative treatment and medical & \\
\hline Physical therapy & 575 \\
\hline Occupational therapy & 261 \\
\hline Prosthetics and orthotics & 196 \\
\hline Casting (Ponsetti serial casting, single casting, hip spica, etc.) & 145 \\
\hline Speech and language pathology & 62 \\
\hline Assistive technology: walking devices, wheelchairs & 8 \\
\hline Medical treatment & 31 \\
\hline
\end{tabular}

The numbers reflect single interventions, except for casting, occupational therapy, physical therapy, and speech and language pathology

\section{Committed partners}

The greatest challenge is socioeconomic in nature. In addition to medical costs, patients face many hidden expenses related to transportation, lodging, food, and loss of income [4, 11]. Without financial assistance, many families will choose not to pursue treatment, thus prolonging the burden of disease and perpetuating the cycle of poverty.
Because the project focuses on vulnerable families, it is necessary for the cost of the program to be provided by an external charitable organization to guarantee sustainability. Despite having a sustained funding stream over the past 15 years, SDSS has diversified their income sources and projects beyond the CHRCP to strengthen their sustainability, including engagement with the local and national government services. Additionally, the SDSS staff have been in discussion with the Nepali Ministry of Health to explore ways of building the sustainability of the program by integrating patient navigation into the national health strategic plan.

\section{District level capacity-building}

District hospitals serve a vital role in a country's healthcare, despite this role having oftentimes been neglected. Empowering physicians improves interfacility referrals, a key factor in reducing delays in accessing care [26-28]. This project aims to encourage capacity building at the district level in rehabilitation medicine and pediatric cardiology through bridging the gap between remote facilities and tertiary care centres.

\section{Counselling}

In remote areas, the absence of locally available medical infrastructure forces people to travel despite dangerous conditions or to forego the required treatment altogether [11]. Travel occurs only once all local resources have been exhausted, including visits to traditional healers and health posts, and after desperate agrarian families have sold land and livestock to cover previous treatment. Most families travel on foot to reach the sending facility, with their child suspended in a sling, or carried on a family member's back [29]. They believe the remote clinic will be the last stop for definitive care. Hearing they are required to travel further to Kathmandu presents an insurmountable burden. Effective counselling at the sending facilities is essential to convince families to accept additional travel to receive care [28].

Patients from vulnerable people groups commonly have less access to education and therefore cultural beliefs, local traditions, and opposing advice from traditional healers impact their access to medical care [11]. Overcoming health illiteracy is complicated and multifactorial. It requires extensive individual counselling.

Counselling also plays an important role in accepting life-altering surgery. The patient navigators request a second opinion when medical recommendations do not match 


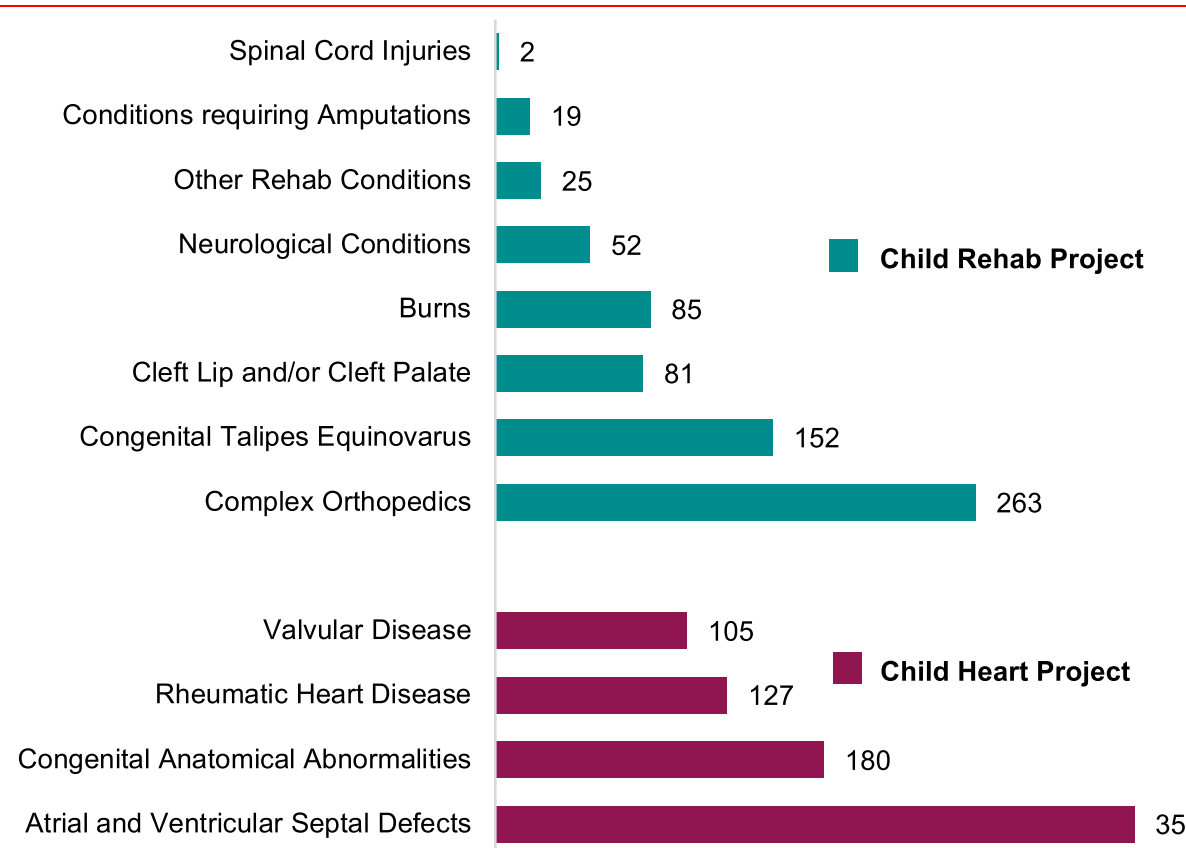

Fig. 3 Patient load per diagnosis category 2006-2018

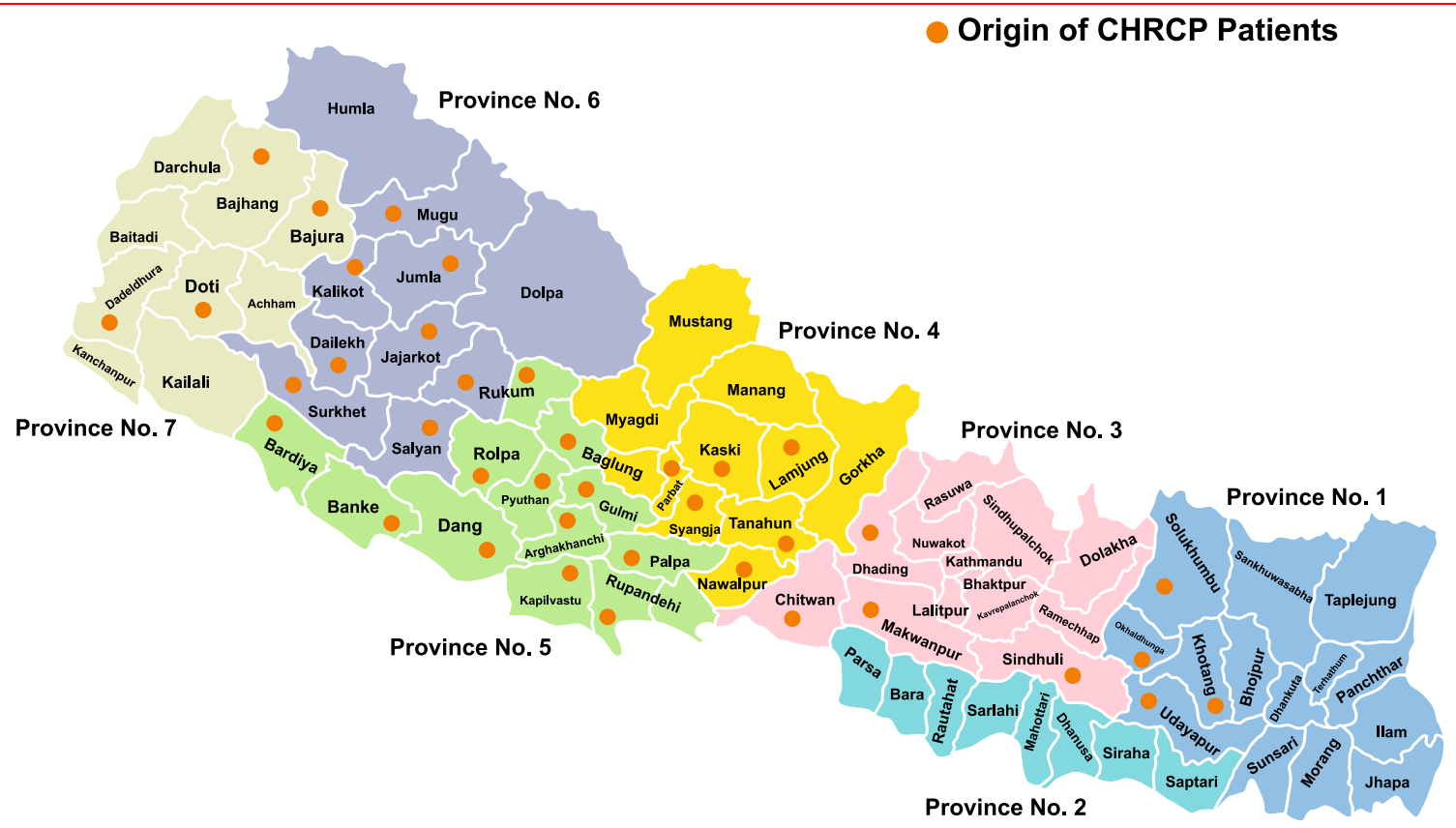

Fig. 4 Origin of CHRCP patients

previous experience. This proactive advocacy has avoided several cases of unnecessary amputations.

Over time, the severity of patient conditions being admitted to the project increased. The patient navigators became adept at helping families understand when no further treatment was possible, assisting them through heart-breaking disappointment and encouraging them to accept a potential long-term disability. Occasionally, a patient in critical condition passed away. Patient navigators provided tremendous support to grieving families by 
Table 4 Recurring challenges and solutions

\begin{tabular}{ll}
\hline Challenges & Solutions \\
\hline Patient adherence & Patients show initiative by setting out to access the referring hospital for care \\
If the patient arrives as an outpatient, the patient and caregiver are advised to immediately set off for \\
Kathmandu the next day, avoiding a return home. If they return home to settle their affairs, they often do \\
not return. The multidisciplinary team emphasizes the need to act quickly, avoiding any unnecessary \\
returns to the village \\
If the patient is coming from inpatient care, the referral is planned in advance and the family receives prior \\
counselling \\
Flexibility is given as to who might be a caregiver: a grandparent, aunt/uncle, or sibling can accompany the \\
patient to Kathmandu so that the parent can stay to provide income for the family or care for other \\
children \\
Patient consent for navigation is obtained upon arrival at the PN centre \\
$\begin{array}{l}\text { Legal implications } \\
\text { Mental health challenges related } \\
\text { to disability }\end{array}$ \\
$\begin{array}{l}\text { Blood shortage } \\
\text { Return to home village }\end{array}$ \\
$\begin{array}{l}\text { Involvement in community life at SDSS gives birth to hope after disability } \\
\text { Adaptation of home environment through local NGO }\end{array}$
\end{tabular}

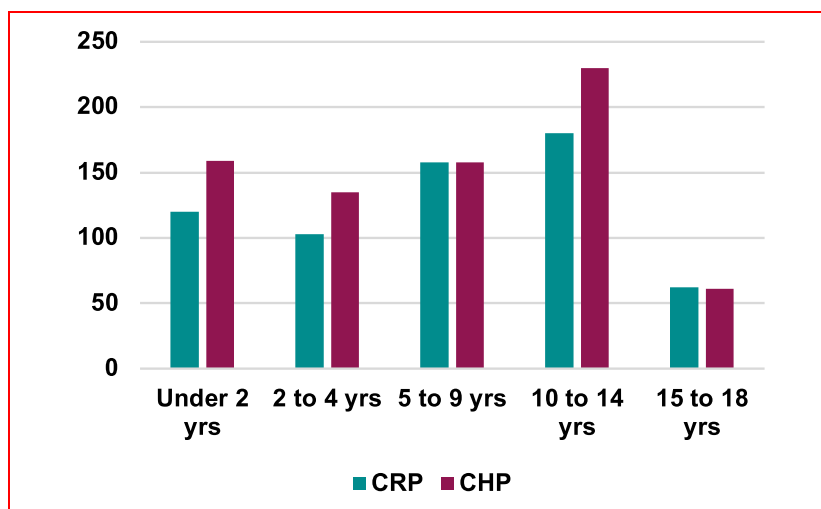

Fig. 5 Age distribution of patients over the 12-year study period

walking through the tragedy alongside them, assisting with legal paperwork, initiating funeral procedures, and securing transportation home.

\section{Advocacy and expertise}

Social marginalization is particularly evident when patients from remote villages or low social status must travel to a large metropolitan city, where illiteracy and language differences add further barriers to accessing care within the chaos of an overcrowded and sometimes dangerous environment. Patients and families from higher social classes tend to confidently demand and obtain services, whereas people from lower social classes and rural areas generally lack these skills [30, 31].
Interestingly, in situations when financial burden is not a problem, cultural barriers can still prevent access to care [11] and require advocacy on the part of the SDSS staff.

SDSS' patient navigators have become experts with the hospital policies and procedures, granting them access to a variety of support programs offered by the government and other NGOs. They maintain current understanding of the services available at the multiple Kathmandu hospitals. Medical staff located at remote sending hospitals rely on the patient navigators' knowledge, consulting them before making a formal referral.

\section{Compassion and community}

SDSS has a no-refusal policy. This impacts the overall budget of the organization, while giving them a reputation for being compassionate. Indeed, the Kathmandu tertiary hospitals have grown to respect the SDSS services and often call upon them to assist with patients already admitted through other channels.

Nepal still grapples with significant stigma and prejudice surrounding disability. Many of the SDSS staff are persons living with disabilities, and therefore can demonstrate the potential for a positive future, offering hope to families who are struggling with a new diagnosis of permanent disability. Patients and their families develop supportive relationships within the SDSS community both during their stay and over scheduled follow-up visits.

The SDSS patient navigation model is unique in that it spans the country and functions as an interfacility system, linking remote sending facilities to a variety of specialized 
tertiary care facilities through a central patient navigation hub.

Present plans for expansion involve improving early intervention at the community level by accepting referrals from community-based rehabilitation facilities for select diagnoses.

\section{Conclusion}

The comprehensive patient navigation model at SDSS is effective in overcoming many of the barriers faced by vulnerable patients in accessing appropriate tertiary facilities. It bridges the gaps in the existing healthcare system.

As humanity faces the new challenge of lockdowns to control COVID-19, the added barriers to access routine healthcare will inevitably lead to increases in morbidity and mortality [32, 33]. More than ever before, countries must maintain a high priority in assisting the most vulnerable as we strive to deliver Universal Health Coverage.

Patient navigation through an efficient interfacility referral system is an innovative strategy to reach some of the most remote and poverty-stricken places on earth. We believe that replicating this model would benefit patients in overcoming barriers to access surgical care and rehabilitation in other LMIC settings.

Acknowledgements The authors wish to acknowledge that this project was birthed through the compassionate initiative of Sundar Dhoka church, who courageously started up the work of navigating patients through Kathmandu. We would like to thank Samaritan's Purse for their guidance, partnership and funding of this project from the initial phase until the present day.We would also like to express our thanks to Jeanie Ough for editing the manuscript, and to Dr. Wim Brandsma for critically reviewing our work.

Funding This study did not receive funding.

\section{Declaration}

Conflict of interest The authors declare that they have no conflict of interest.

Informed consent Informed consent was obtained from all individual participants in the program.

Extra Web Material For more information on the patient navigation program at Sundar Dhoka Saathi Sewa: https://vimeo.com/23621796

Open Access This article is licensed under a Creative Commons Attribution 4.0 International License, which permits use, sharing, adaptation, distribution and reproduction in any medium or format, as long as you give appropriate credit to the original author(s) and the source, provide a link to the Creative Commons licence, and indicate if changes were made. The images or other third party material in this article are included in the article's Creative Commons licence, unless indicated otherwise in a credit line to the material. If material is not included in the article's Creative Commons licence and your intended use is not permitted by statutory regulation or exceeds the permitted use, you will need to obtain permission directly from the copyright holder. To view a copy of this licence, visit http://creativecommons. org/licenses/by/4.0/.

\section{References}

1. World Health Organization (2012) Violence, injuries, and disability: biennial report 2010-2011. WHO 3-14

2. World Health Organization (2014) Injuries and violence: the facts 2014. WHO 2-10

3. Department of Economic and Social Affairs. Disability and development report 2018. United Nations (2019) 1-22, 27, 31-46, 47-63

4. Chan M, Zoellick RB (2011) World report on disability 2011. WHO and World Bank ix-xi, 25, 62-92, 94-108.

5. World Health Organization (2008) Violence, injuries and disability, biennial 2006-2007 report. WHO 4

6. United Nations Development Program. Sustainable Development Goals. https://www.undp.org/content/dam/undp/library/corpo rate/brochure/SDGs_Booklet_Web_En.pdf

7. World Health Organization (2019) Rehabilitation in health systems: guide for action. WHO 2-3

8. Meara JG, Leather AJ, Hagander L et al (2015) Global Surgery 2030: evidence and solutions for achieving health, welfare, and economic development. Lancet 386:569-624

9. Kim JY. Speech by World Bank Group President Jim Yong Kim on Universal Health Coverage in Emerging Economies. http:// www.worldbank.org/en/news/speech/2014/01/14/speech-worldbank-group-president-jim-yong-kim-health-emerging-economies

10. Shrime MG, Dare A, Alkire BC et al (2016) A global countrylevel comparison of the financial burden of surgery. BJS 103:1453-1461

11. Grimes CE, Bowman KG, Dodgion CM et al (2011) Systematic review of barriers to surgical care in low-income and middleincome countries. World J Surg 35:941-950

12. United Nations Development Program (2016) Human development report 2016. UNDP 60:197-219

13. Asian Development Bank Country Partnership Strategy (2013-2017) Country poverty analysis: Nepal (detailed). https:// www.adb.org/sites/default/files/linked-documents/cps-nep-20132017-pa-detailed.pdf

14. Nepali S, Simkhada P, Davies I (2019) Trends and inequalities of stunting in Nepal: a secondary data analysis of four Nepal demographic health surveys from 2001 to 2016. BMC Nutr 5(1):1-10. https://doi.org/10.1186/s40795-019-0283-x

15. The World Bank. Nepal Data. https://data.worldbank.org/coun try/nepal

16. Yadava YP. Language use in Nepal (2014) Population monograph of Nepal, Kathmandu, Central Bureau of Statistics. Volume II, Chapter 2, 51-72. https://nepalindata.com/resource/popula tion-monograph-of-nepal-volume-ii-social-demography/

17. Thapa R, Bam K, Tiwari P et al (2019) Implementing federalism in the health system of Nepal: opportunities and challenges. Int $\mathrm{J}$ Health Policy Manag 8(4):195-198

18. Ministry of Health and Population (2014) Human resources for health, Country Profile, Nepal 10-11

19. Scovil CY, Ranabhat MK, Craighead IB et al (2012) Follow-up study of spinal cord injured patients after discharge from inpatient rehabilitation in Nepal in 2007. Spinal Cord 50:232-237

20. Ghimire P (2014) Nepal may have enough doctors but they're in the wrong place. BMJ 349:g4913

21. Maru D, Pierson J, King C (2013) Himalayan health: improving hospitals and clinics in western Nepal. The Solut J 4:59-65 
22. Nepal Physiotherapy Association. The significant shift in Nepal's rehabilitation sector after the recent earthquake: a report on Nepal earthquake and rehabilitation responses. Kathmandu. http:// nepalphysio.org.np/wp-content/uploads/2015/10/NEPTA-reporton-Nepal-Earthquake.pdf

23. Paudel YR, Dariang M, Keeling SJ et al (2026) Addressing the needs of people with disability in Nepal: the urgent need. Disabil Health J 9:186-188

24. Nagarajan N, Gupta S, Shresthra S et al (2015) Unmet surgical needs in children: a household survey in Nepal. Pediatr Surg Int 31:389-395

25. Gupta S, Ranjit A, Shrestha R et al (2014) Surgical Needs of Nepal: Pilot study of population based survey in pokhara. Nepal World J Surg 38:3041-3046

26. Yeoh ZY, Jaganathan M, Rajaram N et al (2018) Feasibility of patient navigation to improve breast cancer care in Malaysia. $\mathrm{J}$ glob oncolog 4:1-13

27. Rajbhandari R, McMahon DE, Rhatigan JJ et al (2020) The neglected hospital - the district hospital's central role in global health care delivery. N Engl J Med 382(5):397-399

28. Pouramin P, Li CI, Busse JW et al (2020) Delays in hospital admissions in patients with fractures across 18 low-income and middle-income countries (INORMUS): a prospective observational study. Lancet Glob Health 8:e712-720

29. Gurung TD (2015) Boy walked 8 days to treat broken arm. Nepali Times, Kathmandu, Himalmedia Pvt Ltd

30. Raut A, Thapa P, Citrin D et al (2015) Design and implementation of a patient navigation system in rural Nepal: Improving patient experience in resource-constrained settings. Healthcare (Amst) 3:251-257

31. Matousek AC, Addington SR, Kahan J et al (2017) Patient navigation by community health workers increases access to surgical care in Rural Haiti. World J Surg 41:3025-3030

32. Roberton T, Carter E, Chou VB et al (2020) Early estimates of the indirect effects of the COVID-19 pandemic on maternal and child mortality in low-income and middle-income countries: a modelling study. Lancet Glob Health 8(7):e901-908

33. Siddiqua A, Bhandari M (2020) Impact of COVID-19 lockdowns in the developing world: a humanitarian crisis like no other. Orthoevidence Insights, Article No, p 20

Publisher's Note Springer Nature remains neutral with regard to jurisdictional claims in published maps and institutional affiliations. 